\title{
Accessibility, Usability, Safety, Ergonomics: Concepts, Models, and Differences
}

\author{
Klaus Peter Wegge ${ }^{1}$ and Dirk Zimmermann ${ }^{2}$ \\ ${ }^{1}$ Siemens Business Services C-LAB, Fürstenallee 11, 33102 Paderborn, Germany \\ ${ }^{2}$ T-Mobile Deutschland GmbH, Landgrabenweg 151, 53227 Bonn, Germany \\ klaus-peter.weggeac-lab.de, dirk.zimmermannat-mobile.de
}

\begin{abstract}
The purpose of this paper is to clearly point out commonly agreed definitions of the terms Ergonomics, Usability, Accessibility and Safety, their relations to each other, overlaps and differences and their influence on the design of products and services.
\end{abstract}

Keywords: Accessibility, Ergonomics, Usability, Safety; Differences between Accessibility \& Usability.

\section{Introduction}

The purpose of this paper is to clearly point out commonly agreed definitions of the terms Ergonomics, Usability, Accessibility and Safety, their relations to each other, overlaps and differences and their influence on the design of products and services .

It is well recognized that these different terms are often mixed up unintentionally and replaced by synonyms. "Terms such as design for all, barrier-free design, inclusive design and transgenerational design are used similarly but in different contexts" (ISO/IEC Guide 71, chapter 3.2. Note 1 [2]). Also terms like "Universal Design", "Universal Accessibility" or "Accessibility for All" belong to this category, underlining the intention that products "are usable by all people, to the greatest extent possible, without the need for adaptation or specialized design" (ISO/IEC Guide 71, chapter 3.2. Note 2 [2]). A precise definition of these synonyms is often not available. Therefore they are not covered by this paper.

Further confusions arise from the word "Usability" which is mostly used in the sense of the basic "use" or "usage" of products by the user (e.g. ISO/IEC Guide 71, chapter 1.1.A [2]) but not as a terminus technicus for a design concept called "Usability" which is defined in ISO 9241-11 [5] (see chapter 2.2.).

The intention of this paper is therefore to stimulate a consistent use of the terms Accessibility, Usability, Safety and Ergonomics e.g. in standards, guidelines and regulation and to avoid unnecessary confusion and discussions of stakeholders.

\section{Terminology}

The concepts of Accessibility, Usability and Safety all have a foundation in or relation to the area of Ergonomics. However, they all have a different focus in their specific area e.g. user groups, requirements, methods and legal implication (see Figure 1). 


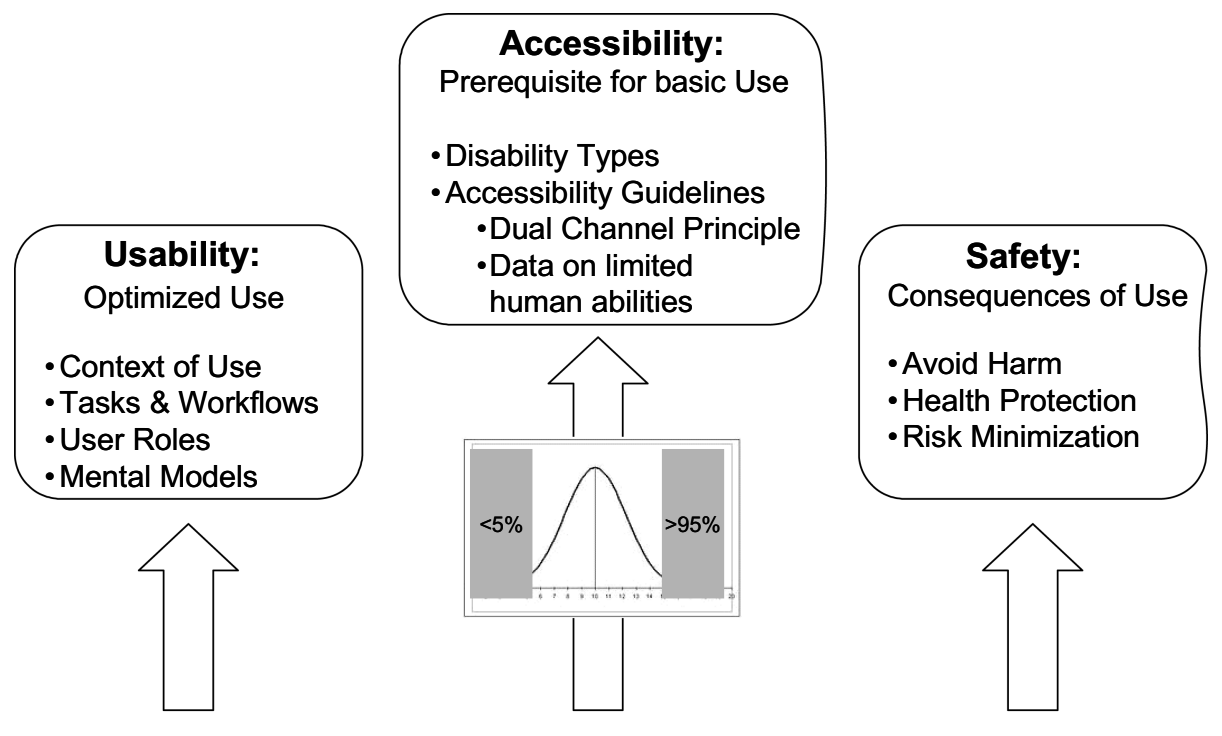

Ergonomics: Product Design

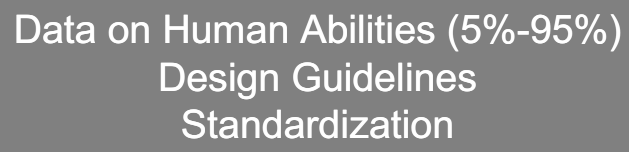

Fig. 1. Accessibility, Usability and Safety in relation to Ergonomics

\subsection{Ergonomics}

DIN/EN/ISO 6385 [9] defines Ergonomics as a "scientific discipline concerned with the understanding of interactions among human and other elements of a system, and the profession that applies theory, principles, data and methods to design in order to optimize human well-being and overall system performance".

Ergonomics deals with general design principles, providing design guidance by using data on human work performance. "Properly applied, ergonomics optimizes the performance and effectiveness of the work system, including the workers, without detriment to their health, well-being or safety." (DIN/EN/ISO 6385 [9])

The three areas of Accessibility, Usability and Safety can be seen as specialized facets of Ergonomic Design

\subsection{Accessibility}

ISO/IEC Guide 71 [2], which is equal to CEN/CLC Guide 6, defines Accessible Design as "design focused on principles of extending standard design to people with 
some type of performance limitation to maximize the number of potential customers who can readily use a product, building or service".

Therefore it "widens the scope of users as far as possible" and "is not limited to the 5th to 95th percentiles of working populations." (ISO DTR 22411 [3])

Accessibility is a prerequisite for basic use of products by elderly persons and persons with sensory, physical or cognitive disabilities. Generally, the Usability criteria Effectiveness, Efficiency, don't play any practical role in accessible design yet, let alone Satisfaction.

There are three main strategies (see ISO/IEC Guide 71, Chapter 3.2 [2]) to achieve accessible products:

1. "designing products, services and environments that are readily usable by most users without any modification," (Universal Design)

2. "by making products or services adaptable to different users (adapting user interfaces)," (Adaptive Design)

3. "and by having standardized interfaces to be compatible with special products for persons with disabilities." (Interoperability with Assistive Technology).

The European Commission prefers a similar definition, but uses Design for All (DFA) as a term instead of Accessible Design:

"There are three main strategies for DFA:

1. design for most users without modifications,

2. design for easy adaptation to different users (e.g. using adjustable interfaces),

3. design with a view to connect seamlessly to assistive devices." (COM2005/425 [10])

ISO 9241-171 [7] and 9241-20 [6] define Accessibility in a very different way as "usability of a product, service, environment or facility by people with the widest range of capabilities".

"The concept of accessibility addresses the full range of user capabilities and is not limited to users who are formally recognized as having a disability."

"The usability-orientated concept of accessibility aims to achieve levels of effectiveness, efficiency and satisfaction that are as high as possible considering the specified context of use, while paying particular attention to the full range of capabilities within the user population." The mixture of the concepts of Accessibility and Usability may occur for historical reasons. But it is remarkable that even in the same standardization organization incompatible definitions are used.

\section{Universal Design}

The first approach of Accessible Design provides ergonomic data on the limited abilities of elderly persons and persons with sensory, physical or cognitive disabilities, aiming at including the widest possible range of user abilities (see ISO/IEC Guide 71, chapter 4.2.1 [2]).

This general data are e.g. provided in section 9 of ISO DTR 22411 [3]. This approach should however be limited in its application, in order to avoid disadvantages to the majority of the user group and discrimination against or stigmatization of elderly persons and persons with disabilities [13]. 
Additionally, conflicting requirements may occur from the different types of disabilities.

\section{Adaptive Design}

The second approach of Accessible Design provides design techniques for "compensation for impaired abilities with alternative modality(ies). This approach is called alternative format" (see ISO/IEC Guide 71, chapter 4.2.1 [2]) and is also known as Dual Channel Principle. Adaptive Design aims at allowing users to adapt the product or service to their specific individual needs (e.g. switch off the child safety lock for one hand operation). These design techniques are e.g. provided in section 8 of ISO DTR 22411 [3] (while not interfering with the majority of users). Very initial recognition of the effectiveness of use of alternative modalities can be seen in the area of software accessibility.

\section{Interoperability with Assistive Technology}

The third approach is Interoperability with Assistive Technology; allowing users with special needs to utilize their commonly used assistive devices (e.g. screen readers, wheel chairs, and hearing aids). A prerequisite for interoperability is often the support of standard interfaces by the product.

\subsection{Usability}

ISO 9241-11 [5] defines Usability as "Extent to which a product can be used by specified users to achieve specified goals with effectiveness, efficiency and satisfaction in a specified context of use."

Usability deals with methods to assess the effective, efficient and satisfactory use of a product in a given context of use, i.e. looks at products in regard to their support for different roles, usage scenarios, tasks or workflows.

To transfer this usability methods to the concept of Accessible Design with its three main strategies is not possible e.g. because of the wide range of different disabilities and experience of users with disabilities and their familiarization with assistive technologies.

\subsection{Safety}

ISO/IEC Guide 51 [1] defines Safety as "Freedom from unacceptable risk. The area of Safety is concerned with the intended or unintended consequences of using a product, e.g. health risks, environmental protection, etc.

Safety requirements apply to the intended user group of the product. There is a tendency to extend the user group to all users, regardless of ability or age (i.e. including children or elderly persons).

\section{Implementing Accessibility, Usability and Safety}

There are usually very different reasons for implementing the three different principles into product design. 


\subsection{Why Companies Implement Accessibility}

Accessibility, especially in the fields of ICT, buildings and public transport, is mandated by national laws, supported by international and national standards and guidelines, and stimulated by public procurement in many countries. Besides this, many companies strive to implement Universal Design in their mainstream products to address a wider market.

There are currently models for labeling and (third-) party certification in the field of accessibility, which are under discussion and, in some countries, in development. With the public procurement procedure required by the US rehabilitation act section 508 , self declaration of conformity by the bidders is the established method for a number of years now.

A company has to consider all of these facts during the product design ideally from the very early beginning. Because of a missing international harmonisation, the resulting requirements lead to market fragmentation for companies that are active on a global market. Examples for laws and regulation in the area of Accessibility:

- the Americans with Disabilities Act,

- $\quad$ the US Telecomm. Act Section 255,

- the US Rehabilitation Act Section 508,

- $\quad$ the EU Mandate 376 (M376, 2006),

- the EU Anti Discrimination Directives,

- $\quad$ the EU telecomm. Directives, and

- $\quad$ "Behinderten Gleichstellungs-Gesetz" in Germany.

\subsection{Why Companies Implement Usability}

In contrast to Accessibility, Usability is often considered a competitive advantage. With technology being more and more a commodity that is taken for granted, the market demand for Usability is increasing. The implementation of usability is voluntary, i.e. usually happens for business reasons and is rarely mandated by laws and regulations. The few existing laws (e.g. the BildschArbV in Germany) are vague in the area of usability and cannot easily be tested against.

Because of the underlying economic motives, the success of usability engineering is often monitored through tests, market success or Return-on-Investment analyses. Conformity with standards is not seen as a valuable selling point, therefore standards and certification (e.g. according to ISO 9241 [4] or ISO FDIS 20282 [8]) only play a secondary role for manufacturers.

\subsection{Why Companies Implement Safety}

Safety is mandatory for the introduction of products, i.e. no product can be marketed without legal consequences if it doesn't meet safety regulation and standards of target market (e.g. CE sign).

\section{Evaluation Strategies}

In order to test against requirements in the different areas described in this paper, two distinctly different approaches can be taken, i.e. user or expert based. For each of the 
areas, there is usually a set of different usage types (e.g. different users, different settings, etc.), and the goal of user based testing is to utilize participants covering the different usage types to elicit feedback on the solution with regard to the set of requirements.

The focus here is on whether the product supports the specific usage types, and if the system model of the product matches the mental model of the users.

Expert based testing has a different focus, i.e. it mostly focuses on conformity with standards, as well as general / generic requirements for and expert knowledge of the specific area.

The evaluation can span different usage types, but will usually not yield as detailed requirements as a user based test. It does, however, usually produce more overarching results that cross usage types, which may not be as easy to elicit from representatives of a single usage type.

\subsection{Accessibility Evaluation}

In the area of Accessibility Evaluation, the different classes of disability constitute the usage types. There are different requirements depending on e.g. whether the user is physically or cognitively impaired. In the evaluation, representatives for each group provide feedback on whether the product supports their specific disability type, if the product fits their mental model, if it is interoperable with the assistive devices they commonly use, and if the product supports certain compensation strategies they have learned to cope with their impairment.

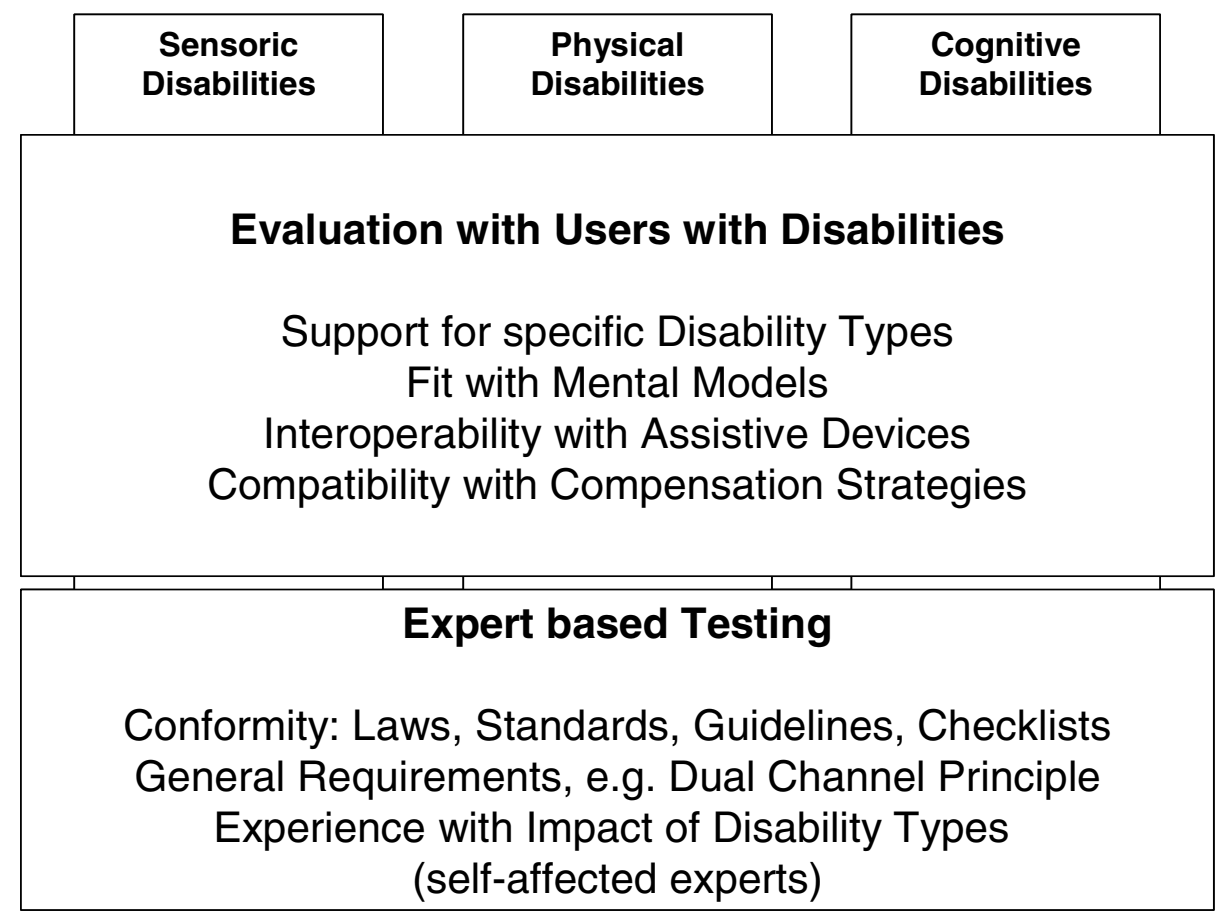

Fig. 2. Accessibility Evaluation - Focus on Disability Types 
Expert based evaluation focuses on overarching aspects. Conformity with current laws, standards, guidelines and checklists is one, testing against general requirements like e.g. the Dual Channel Principle is another.

If experts are self-affected, they can also provide a broader view on the impact of certain disabilities that goes beyond the pure user role.

\subsection{Usability Evaluation}

For evaluating Usability, the usage types are usually defined by different contexts of use. These contexts can either be based on the type or role of the user, or the specific setting (physical, organizational, etc.) the task is carried out in. Again, the evaluation focuses on the support for the different contexts of use and fit with the mental model, but also for compatibility with general learning or problem solving strategies of the users. The DIN/EN/ISO 9241 [4] Framework for Usability Evaluation uses Effectiveness, Efficiency and Satisfaction as evaluation criteria.

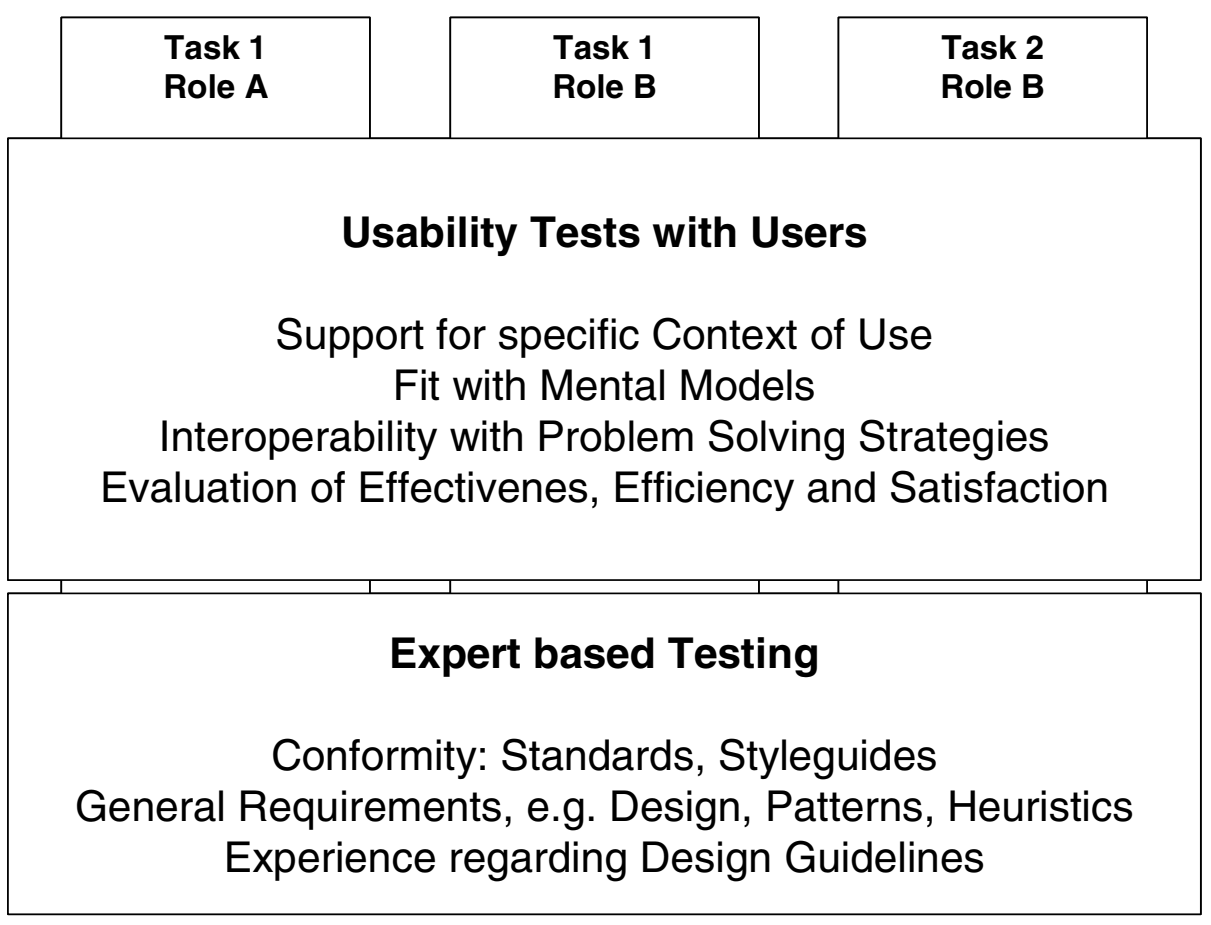

Fig. 3. Usability Evaluation - Focus on Contexts of Use

An expert based evaluation in the area of usability focuses on standard and style guide conformity and commonly known requirements, e.g. patterns or heuristics. It utilizes the expertise on design guidelines to evaluate against those as well. 


\section{Summary and Outlook}

The accessibility of products and services is an absolute prerequisite for the inclusion of elderly persons and persons with disabilities in our modern information and communication society. Currently there are strong efforts on both, the political and industry's side to support this important goal. The started process only can be economically reasonable and really helpful for elderly and disabled persons when regulation and supporting accessibility standardization will follow an international harmonized approach. This is very important because companies are producing for a world market and the needs of persons with disabilities are nearly the same in all countries. Therefore both authors strongly recommend from their professional accessibility experience to avoid national or local solutions and fragmentation in the field of accessibility. As standards play an important role in the field of accessibility, a consistent use of terms and definitions will support implementing accessibility. Contradictory terms and requirement should be avoided to make the concept of accessibility successful, all over the world.

\section{References}

1. ISO/IEC Guide 51. Safety Aspects - Guidelines for their inclusion in Standards. International Organization for Standardization (1999)

2. ISO/IEC Guide 71. Guidelines for standards developers to address the needs of older persons and persons with disabilities. International Organization for Standardization (ISO) (2001)

3. ISO/AWI TR 22411 Ergonomic data and guidelines for the application of ISO/IEC Guide 71 in standards related to products and services to address the needs of older persons and persons with disabilities, (2004)

4. DIN EN ISO 9241. Ergonomic requirements for office work with visual display terminals (VDTs). International Organization for Standardization (1998)

5. DIN EN ISO 9241-11. Ergonomic Requirements for Office Work with Visual Display Terminals, Part 11, Usability (Principles) (1998)

6. DIN EN ISO 9241-20. Ergonomics of human-system interaction - Part 20: Accessibility guidelines for information/communication technology (ICT) equipment and services (2006)

7. DIN EN ISO 9241-171. Ergonomics of human-system interaction - Part 171: Guidance on software accessibility (2006)

8. DIN EN ISO 20282. Ease of operation of everyday products (2006)

9. DIN EN ISO 6385. Ergonomic principles in the design of work systems (2004)

10. COM 2005/42. Communication from the Commission to the Council, the European Parliament and the European Economic and Social Committee and the Committee of the regions, 13.9.2005

11. PRM TSI. Technical Specification for Interoperability - Accessibility for People with Reduced Mobility

12. European Commission M376. Standardization mandate to CEN, CENELEC and ETSI in support of European Accessibility requirements for public procurement of products and services in the ICT Domain

13. State University of North Carolina, Trace Center. The Principles of Universal Design 